\title{
ChemComm
}

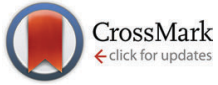

Cite this: Chem. Commun., 2015, 51, 7112

Received 21st February 2015, Accepted 18th March 2015

DOI: $10.1039 / \mathrm{c5cc01583a}$

www.rsc.org/chemcomm

\section{Hyper-expanded interlayer separations in superconducting barium intercalates of $\mathrm{FeSe} \uparrow$}

\author{
K. V. Yusenko, ${ }^{a b}$ J. Sottmann, ${ }^{b}$ H. Emerich, ${ }^{c}$ W. A. Crichton, ${ }^{d}$ L. Malavasi ${ }^{e}$ and \\ S. Margadonna*a
}

\begin{abstract}
Intercalation of $\mathrm{Ba}$ in $\beta$-FeSe by ammonothermal synthesis results in the formation of different superconducting phases with interlayer distance ranging between 8.4 and $13.1 \AA$. The values of $T_{\mathrm{c}}$ are primarily dependent on $\mathrm{Ba}$ content, and are further modulated by the interlayer spacing through facile intercalation and deintercalation of ammonia.
\end{abstract}

Among the various families of Fe-based superconductors, layered tetragonal $\beta$-FeSe with transition temperature $T_{\mathrm{c}}=8 \mathrm{~K}$ has attracted tremendous interest. ${ }^{1}$ It was immediately recognized that this system offers unique opportunities to study the pairing mechanism due to its apparent chemical and structural simplicity. Furthermore, the superconducting transition is extremely sensitive to applied pressure, reaching the value of $39 \mathrm{~K}$ at $7 \mathrm{GPa}^{2}$ Intercalation of guest species in between the layers is also generating much attention following the discovery of superconductivity, at about $30 \mathrm{~K}$, in the $\mathrm{A}_{x} \mathrm{Fe}_{2-y} \mathrm{Se}_{2}(\mathrm{~A}=\mathrm{K}, \mathrm{Rb}, \mathrm{Cs}$ and $\mathrm{Tl})$ systems. It is now well-established that high temperature solid state synthesis of these compounds leads to inhomogeneous samples, microscale phase separation and the creation of Fe vacancies. ${ }^{3}$ As the superconducting state is strongly dependent on the preservation of the full stoichiometry of the FeSe layers, low temperature reactions of pre-synthesized FeSe with a solution of alkali metal dissolved in liquid ammonia or other organic amines such as pyridine and methylamine, have been employed for the isolation of single-phase vacancy free specimens. The so-obtained materials are bulk superconductors with transition temperatures ranging between 30 and $46 \mathrm{~K} . \mathrm{Li}, \mathrm{Na}, \mathrm{K}, \mathrm{Cs}, \mathrm{Rb}, \mathrm{Ca}$, $\mathrm{Ba}, \mathrm{Sr}, \mathrm{Eu}$ and $\mathrm{Yb}$ intercalates were prepared mainly using liquid ammonia as a solvent. ${ }^{4-8}$ Very interestingly, it has been shown that in the case of the alkali metals also other species such as ammonia

\footnotetext{
${ }^{a}$ College of Engineering, Swansea University, SA2 8PP, Swansea, UK. E-mail: s.margadonna@swansea.ac.uk

${ }^{b}$ Department of Chemistry, University of Oslo, 0315 Oslo, Norway

${ }^{c}$ Swiss-Norwegian Beamlines, The European Synchrotron Radiation Facility, Grenoble, 38043, France

${ }^{d}$ The European Synchrotron Radiation Facility, Grenoble, 38043, France

${ }^{e}$ Department of Chemistry and INSTM, University of Pavia, Italy

$\dagger$ Electronic supplementary information (ESI) available: Materials synthesis and experimental methods. See DOI: 10.1039/c5cc01583a
}

and metal-amides reside between the layers. A substantial amount of work has been reported on the intercalation of the alkali metals $\mathrm{Li}$, $\mathrm{Na}$ and $\mathrm{K}$ by the ammonothermal method. In brief, depending on the preparatory conditions, two $\mathrm{K}$ intercalates $\mathrm{K}_{x} \mathrm{Fe}_{2} \mathrm{Se}_{2}\left(\mathrm{NH}_{3}\right)_{y}$ $\left(x \approx 0.3\right.$ and 0.6 ), were isolated (each of $\mathrm{ThCr}_{2} \mathrm{Si}_{2}$-type). The different amount of $\mathrm{K}$ results in phases with varying interlayer spacing and critical temperatures. ${ }^{5}$

In situ powder X-ray and neutron diffraction experiments performed on $\mathrm{Li}$ intercalates show the formation of at least two phases, namely, an ammonia-rich phase $\mathrm{Li}_{0.6}\left(\mathrm{NH}_{2}\right)_{0.2}\left(\mathrm{NH}_{3}\right)_{1.8} \mathrm{Fe}_{2} \mathrm{Se}_{2}$ accommodating a double layer of $\mathrm{NH}_{3}$ molecules between the FeSe slabs with $T_{\mathrm{c}}=39 \mathrm{~K}(P 4 / \mathrm{nmm}$ with $d \approx 9.0 \AA)$ and an ammonia-poor form that can be obtained by evacuation of the ammonia-rich phase and has composition $\mathrm{Li}_{0.6}\left(\mathrm{NH}_{2}\right)_{0.2}\left(\mathrm{NH}_{3}\right)_{0.8} \mathrm{Fe}_{2} \mathrm{Se}_{2}$ ( $\mathrm{ThCr}_{2} \mathrm{Si}_{2}$-type). ${ }^{6}$ This phase has higher $T_{\mathrm{c}}(44 \mathrm{~K})$ and smaller interlayer spacing in comparison with the ammonia-rich phase. Finally, ammonothermal synthesis of $\mathrm{Na}$ intercalated $\beta$-FeSe with varying concentrations of the $\mathrm{Na}-\mathrm{NH}_{3}$ solutions leads to the isolation of three phases, which are essential vacancy free, and display different interlayer spacings, $T_{\text {c }}$, alkali metal and ammonia content. ${ }^{7}$

Intercalation of alkali earth metals (AE) in $\beta$-FeSe has been achieved for $\mathrm{Ba}$ and $\mathrm{Sr}$ using the ammonothermal method at room temperature in an autoclave. ${ }^{8}$ Superconducting $\mathrm{Ba}_{0.8} \mathrm{Fe}_{2} \mathrm{Se}_{2}$ with $T_{\mathrm{c}}=$ $39 \mathrm{~K}$ is reported to crystallise in the $14 / \mathrm{mmm}$ space group without the presence of other guests between the layers. The realisation that $\mathrm{AE}$ intercalation leads to single $\mathrm{ThCr}_{2} \mathrm{Si}_{2}$-type phase, free of $\mathrm{NH}_{3} / \mathrm{NH}^{2-}$ species and without Fe vacancies, calls for further investigations. As such, these materials can be considered ideal systems to study the normal state and superconducting properties of metal intercalated $\beta$-FeSe compounds and to reveal the important parameters (e.g. interlayer spacing, local structure of the $\mathrm{FeSe}_{4}$ tetrahedra and doping level) which are responsible for the strong enhancement of $T_{\mathrm{c}}$.

In the present work, Ba intercalation was performed using condensed ammonia at $195-200 \mathrm{~K}$ in a vacuum gas line (low temperature (LT) reaction) as well as using an autoclave at room temperature (room temperature (RT) reaction) (Fig. S1, ESI $\dagger$ ). Different conditions were explored including various reaction time and stoichiometries for both synthetic procedures (ESI $\dagger$ ). In all 
Table 1 Selected parameters for the Ba intercalates studied in this work. The phase composition shown was estimated by Rietveld refinements of the Xray data. $d$ is the interlayer spacing. Elemental analysis obtained ex situ on Phase $\mathrm{VI}$ resulted in a composition of $\mathrm{Ba}_{0.30} \mathrm{Fe}_{1.64} \mathrm{Se}_{2}$ (see $\mathrm{ESI}$ for details)

\begin{tabular}{lllll}
\hline Phase & $d(\AA)$ & Symmetry & $T_{\mathrm{c}}(\mathrm{K})$ & Conditions \\
\hline$\beta$-FeSe & 5.465 & $P 4 / n m m$ & 8 & \\
Phase I $\mathrm{Ba}_{0.29}\left(\mathrm{NH}_{3}\right)_{0.35} \mathrm{Fe}_{2} \mathrm{Se}_{2}$ & $13.125(1)$ & $I 4 / m m m$ & n.d. & Fast forming intermediate \\
Phase II Ba & $11.527(1)$ & $I 4 / m m m$ & 39 & $<250 \mathrm{~K}$ \\
Phase III Ba $\mathrm{NH}_{3.35}\left(\mathrm{NH}_{3}\right)_{2.12} \mathrm{Fe}_{2} \mathrm{Se}_{2} \mathrm{Se}_{2}$ & $11.089(1)$ & $P 4 / n m m$ & n.d. & $250-280 \mathrm{~K}$ \\
Phase IV $\mathrm{Ba}_{0.37}\left(\mathrm{NH}_{3}\right)_{1.04} \mathrm{Fe}_{2} \mathrm{Se}_{2}$ & $9.060(1)$ & $P 4 / n m m$ & 36 & $>280 \mathrm{~K}$ \\
Phase V & $9.668(2)$ & $P 4 / n m m$ & n.d. & $<280 \mathrm{~K}$, as phase mixtures \\
Phase VI Ba $\mathrm{Ba}_{0.28} \mathrm{Fe}_{2} \mathrm{Se}_{2}$ & $8.363(1)$ & $I 4 / m m m$ & 34 & Vacuum
\end{tabular}

cases, the formation of the same tetragonal phase (space group $I 4 / \mathrm{mmm}$ ) with $d \approx 8.40 \AA$ (Table 1 , Fig. S2, ESI $\dagger$ ) and $T_{\mathrm{c}}=34-35 \mathrm{~K}$ was observed independently of the initial nominal composition.

The structural parameters of the so-obtained samples are in good agreement with those previously reported, even though our experimental $T_{\mathrm{c}}$ is $5 \mathrm{~K}$ lower. ${ }^{8}$ It was then thought, that only one specific polymorph could be stabilized in the $\mathrm{NH}_{3}$-Ba-FeSe phase field. However, when we followed the intercalation reaction in situ using powder X-ray diffraction (Fig. S3, ESI†), a completely different scenario was identified and the formation of a number of different phases was observed (Fig. 1). During the experiments the following protocol was applied: liquid ammonia was condensed at $200 \mathrm{~K}$ in a glass capillary containing both $\beta$-FeSe and Ba which, critically, were not in contact with each other. The AE starts dissolving immediately (to form a 'gold' solution). After a short induction time (10-15 $\mathrm{min})$ the diffraction lines characteristic of $\beta$-FeSe disappear without intermediate amorphisation (Fig. 2) and this is accompanied by the formation of a new phase with a characteristic $d=13.13 \AA$ (Phase I). Phase I is unstable and transforms with time at constant temperature to a new polymorph with smaller interlayer spacing $(d=11.53 \AA)$, which is instead stable at $200 \mathrm{~K}$ (Phase II). The diffraction profiles of both phases can be indexed considering a $I$ lattice and no further extinction conditions

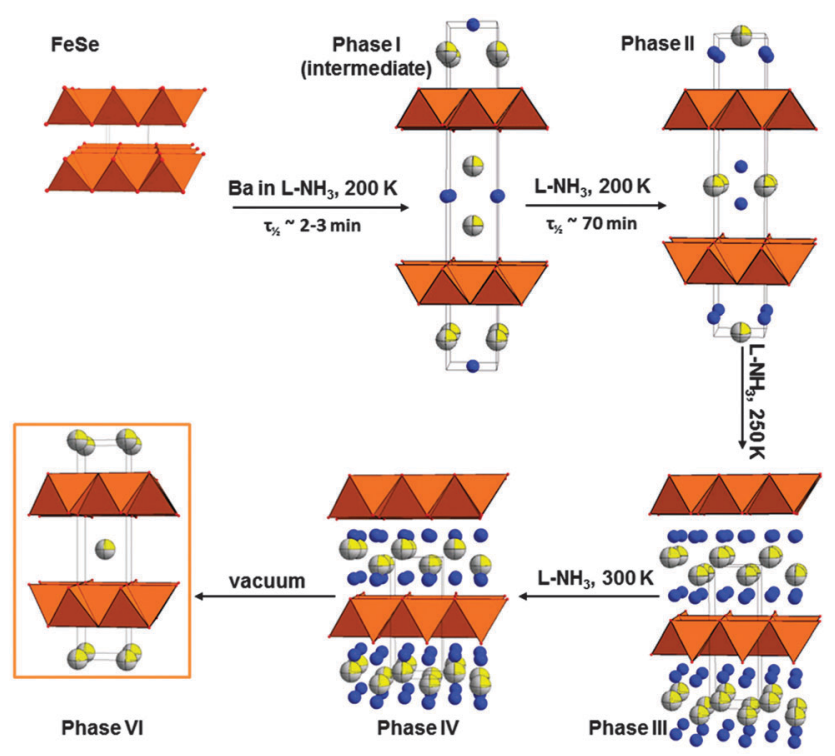

Fig. 1 Schematic representation of the reaction of $\mathrm{Ba}$ and $\beta$-FeSe in liquid ammonia medium at different temperature ( $\mathrm{Ba}$ is shown as grey/yellow spheres, nitrogen - blue). are evident. Time dependence of the evolution of the phase fractions (Fig. 2 and ESI $\dagger$ ) can be fitted as first order with effective constant for the first reaction, $\beta$-FeSe $+\mathrm{Ba} \rightarrow$ Phase I, at least 25-30 times smaller $\left(\tau_{1 / 2} \sim 2-3 \mathrm{~min}\right)$ than that of the following Phase I $\rightarrow$ Phase II $\left(\tau_{1 / 2} \sim\right.$ $70 \mathrm{~min}$ ) transition which is relatively slow. Transformation of Phase I to Phase II can be arrested by cooling the reaction mixture below $195 \mathrm{~K}\left(\mathrm{NH}_{3}\right.$ melting point), which illustrates the overall importance of ammonia in the uptake of interlayer species and phase progression. Sedlmaier $e$ t $a l^{6}$ have investigated Li intercalation into $\beta$-FeSe using in situ diffraction and have not observed any metastable intermediates but only reported the formation of the final ammonia-rich $\mathrm{Li}_{0.6}\left(\mathrm{NH}_{2}\right)_{0.2}\left(\mathrm{NH}_{3}\right)_{1.8} \mathrm{Fe}_{2} \mathrm{Se}_{2}$. Nevertheless, as pointed out, the timedependence of the phase fractions clearly indicate the presence of an intermediate form that, however, could not be identified. It appears then, that intercalation of alkali metals is much faster than that of AE, where instead the formation of the transient phase could be directly observed.

Heating of Phase I and II from 200 to $300 \mathrm{~K}$ initiates several sequential phase transitions: Phase I + Phase II $\stackrel{250 \mathrm{~K}}{\longrightarrow}$ Phase III $\stackrel{250 \mathrm{~K}}{\longrightarrow}$ Phase IV (Fig. 3(a)). Cooling Phase IV in the presence of ammonia results in the formation of phase mixtures. At temperatures lower than $280 \mathrm{~K}$, Phase V + Phase III form while further cooling below $250 \mathrm{~K}$ stabilises

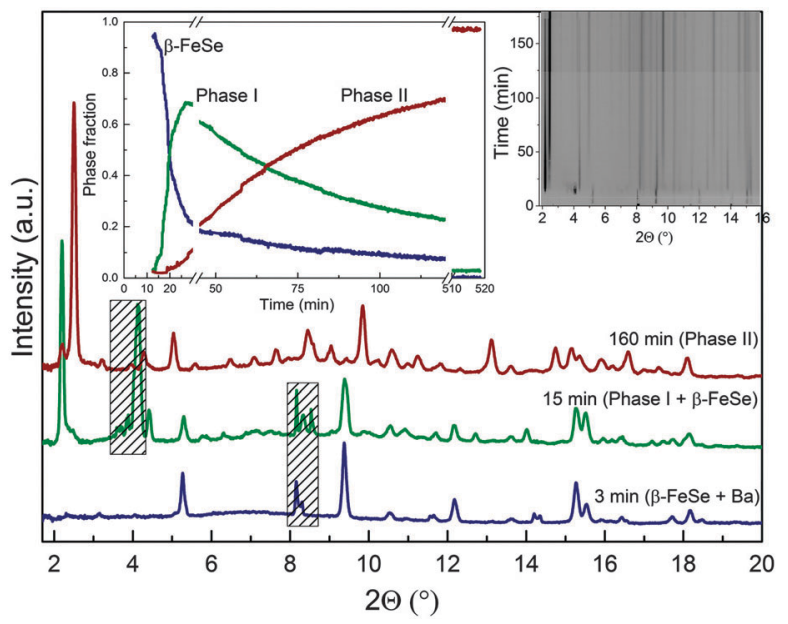

Fig. 2 Diffraction profiles $(\lambda=0.5048 \AA)$ obtained at the initial stage of the reaction between $\mathrm{Ba}$ and $\beta$-FeSe in $\mathrm{NH}_{3}$ at $200 \mathrm{~K}$ (Bragg peaks relative to unknown $\mathrm{Ba}-\mathrm{NH}_{3}$ phases are hatched). Insets: time dependence of the diffraction profiles and phase fractions for $\beta$-FeSe, Phase I and II obtained from Rietveld refinements. 
Phase V + Phase II, which do not undergo any further changes down to $80 \mathrm{~K}$. Phase IV is retained at room temperature but changes into Phase VI upon evacuation. In the absence of ammonia, Phase VI does not transform down to $80 \mathrm{~K}$; while slow cooling in $\mathrm{NH}_{3}$ gas/liquid induces the phase transformation: Phase VI $\rightarrow$ Phase V + Phase III.

Given that these phases are clearly distinguishable by X-ray diffraction and do not represent a continuous series of, e.g. Ba composition, not all phases adopt the same crystal structure (Fig. 3(b)). In the superconducting $\beta$-FeSe metal intercalates, the structures are built from FeSe layers, arranged as $\mathrm{PbFCl}$-like or $\mathrm{ThCr}_{2} \mathrm{Si}_{2}$-like, with included interlayer Ba and ammonia/amide species. Phases I, II, and VI were refined considering a $\mathrm{ThCr}_{2} \mathrm{Si}_{2}$-like basis, while Phases III, IV, and $\mathrm{V}$ adopt structures more-strongly related to the PbFCl-type already reported for the $\mathrm{Li}$ intercalated ammonia-rich phase. ${ }^{6}$ Rietveld refinements were initiated assuming these as initial models. Analysis of differential Fourier maps was performed on all phases apart from $\mathrm{V}$ as the quality of the data in this case does not allow detailed structural investigations. The observed electron density maxima were then associated with intercalated $\mathrm{Ba}$ and $\mathrm{N}\left(\mathrm{NH}_{3}\right.$ and/or $\left.\mathrm{NH}_{2}{ }^{-}\right)$. The results of the Rietveld refinements are summarized in Table 1, Tables S1-S5 and Fig. S5-S9, ESI.†

Phases I and II have the largest interplanar distances among all the experimentally described $\beta$-FeSe systems. In Phase I, Ba and $\mathrm{N}$ partly occupy the available $4 \mathrm{e}$ and $2 \mathrm{~b}$ sites, respectively (Fig. 1).

(a)

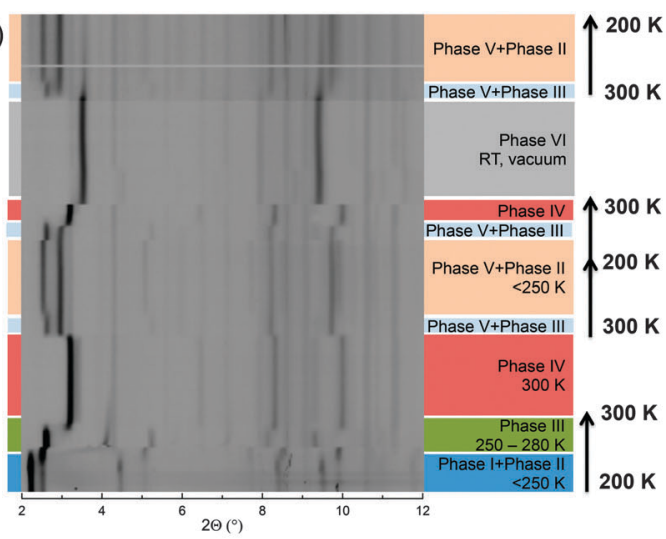

(b)

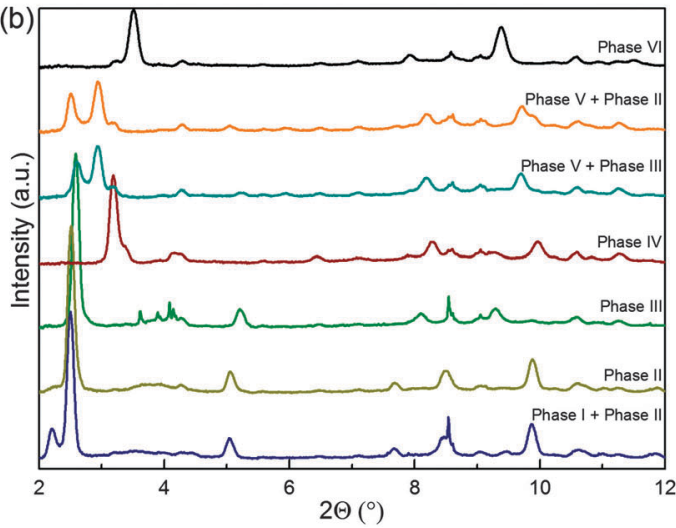

Fig. 3 (a) Waterfall plot of sequential diffraction profiles $(\lambda=0.5048 \AA)$ taken on $\mathrm{Ba}+\beta$-FeSe mixtures on heating, cooling, and upon evacuation; (b) selected diffraction profiles to highlight differences between the isolated phases.
Phase II is very similar with a remarkable shortening of the $c$ axis of 3 $\AA$, which is commensurate with the swapping of the Ba and $\mathrm{N}$ sites, effectively placing the larger $\mathrm{Ba}$ ion distribution in plane with the FeSe layers (Fig. 1). Phase III and IV are PbFCl-like structures and display shorter interlayer spacing due to their crenulated distribution of $\mathrm{Ba}$ and $\mathrm{N}$ sites over the $2 \mathrm{c}$ and $4 \mathrm{f}$ sites. The $\mathrm{N}$ atoms are located above the interstitial positions of the adjacent Se layer. Phase VI is the most simple structure encountered with the sole Ba partly occupying the centre of inversion of the $\mathrm{ThCr}_{2} \mathrm{Si}_{2}$ structure. (Fig. 1). The inclusion of $\mathrm{Ba}$ at this position expands this phase by $\sim 3 \AA$ compared to $\beta$-FeSe. No signature of additional electron density was found in other interstitial sites suggesting that intercalated $\mathrm{N}$ species are either absent or present in small amounts.

The results of our in situ structural investigation clearly show that $\mathrm{Ba}$ intercalation reactions in $\beta$-FeSe are complex and result in the formation of several phases. Some of these are quenchable and, as such, can be further investigated. Particularly important is to couple the values of the interlayer spacings with the superconducting behaviour and $T_{\mathrm{c}}$. Magnetic susceptibility measurements were attempted on samples prepared by quenching reaction mixtures sealed into quartz tubes (ESI $\dagger$ ). Phase VI shows a superconducting transition at $34 \mathrm{~K}$ (Fig. 4). Identical behaviour is displayed by all samples prepared via LT and RT synthesis roots and upon evacuation of Phase IV with typical superconducting fractions $>30 \%$, independently of the starting nominal composition (Fig. S4, ESI $\dagger$ ). "Gold" Ba solution (nearly saturated Ba solution in liquid ammonia) were kept in dry ice and then rapidly inserted into the measurement chamber which was pre-cooled to $200 \mathrm{~K}$. At the very early stage of the reaction, no diamagnetic signal is found while leaving the reaction mixture for $30 \mathrm{~min}$ at this temperature results in the observation of a superconducting transition with $T_{\mathrm{c}}=$ $39 \mathrm{~K}$ (superconducting fraction 28\%). The considerable waiting period, the absence of any signal corresponding to unreacted $\beta$-FeSe and the fact that the superconducting fraction does not change further with time imply that the transition can be related to Phase II. Heating the reaction mixture to room temperature followed by fast quenching below $80 \mathrm{~K}$ results in the immediate appearance of a superconducting phase with $T_{\mathrm{c}}=36 \mathrm{~K}$ which we associate to Phase IV (superconducting fraction $40 \%$ ).

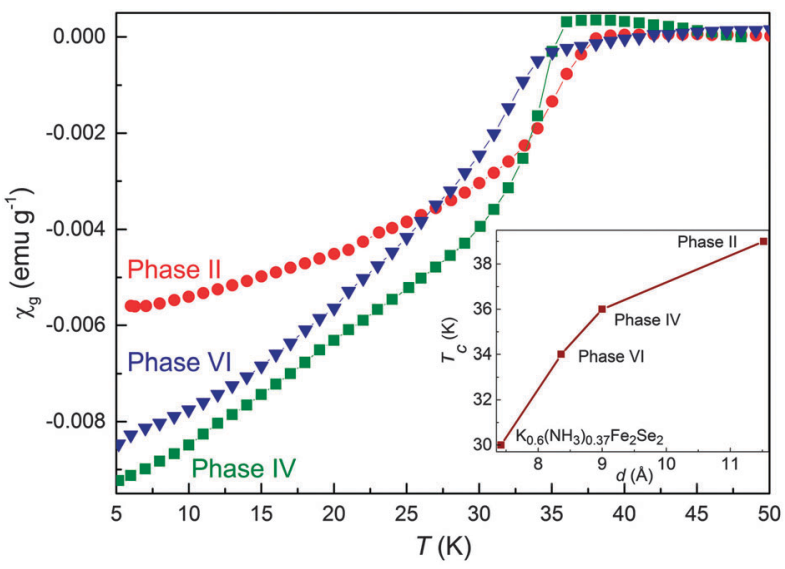

Fig. 4 Zero field cooled magnetization curves for Phases II, IV and VI $(H=10 \mathrm{Oe})$. (inset) Relationship between $T_{\mathrm{C}}$ for and interlayer spacing for $\mathrm{Ba}$ and $\mathrm{K}^{5}$ intercalates on interlayer distances. 
One of the most prominent points arising from our study is that Phase $\mathrm{I}$ to $\mathrm{V}$ can all be described as intermediates of the final product obtained under evacuation of ammonia at $300 \mathrm{~K}$, namely Phase VI. While the different intercalates have distinct interlayer distances ranging between 13.1 and $8.4 \AA$ A, the metal content appears to remain almost constant. The estimated $\mathrm{N} / \mathrm{Fe}$ ratio increases substantially on transformation of Phase I to Phase II, at a rate possibly limited by ammonia supply, to reach a maximum of roughly 1 in Phases II and III followed by a reduction to approximately half of this in Phase IV. The initial nominal composition and concentration of the reaction solutions do not affect the resulting products and only phases with similar Ba content are stabilized. The intercalation/deintercalation of the molecular guest species, which is fundamental for the stabilization of the various phases, is reversible as demonstrated by ammonia-free Phase VI partly transforming back to Phase II in presence of liquid/gas $\mathrm{NH}_{3}$. This provides evidence that the intercalated species are likely to be $\mathrm{NH}_{3}$ molecules as opposed to amide moieties which are more strongly bounded to the metal ion. Further justification for this is given by a nearly constant Fe formal oxidation state of +1.7 , which is consistent with other Fe-based superconductors. ${ }^{7}$ The Fe-Se distances obtained for phases II, IV and VI do not show any significant change and assume values between 2.41 and $2.42 \AA$ which is larger than that of pristine $\beta$-FeSe (2.39 $\AA$ ), providing strong indications of the occurrence of electron transfer between the metal and the FeSe layers. Considering the above discussion, it is plausible to assume that the doping level is essentially invariant throughout the series and, therefore, any further variation of $T_{\mathrm{c}}$ is dependent on other scaling parameters such as interlayer distance, anion height and/or distortion of the $\mathrm{FeSe}_{4}$ tetrahedra. We note that recent theoretical calculations highlight the importance of electron doping as controlling parameter for the value of $T_{\mathrm{c}}{ }^{9}$

The behaviour described above differs from that observed in analogous phases intercalated with the smaller alkali metal $\mathrm{Li}^{+}$and $\mathrm{Na}^{+}$. The first striking difference is that Phase II, which displays the largest $d$-spacing ever observed, has the highest $T_{\mathrm{c}}$ among the $\mathrm{Ba}$ series in contrast with the $\mathrm{Li}$ intercalates where the $T_{\mathrm{c}}$ of the ammonia-rich phase is lower than that of the ammonia-poor. Furthermore, Guo et al. ${ }^{7}$ pointed out that in $\beta$-FeSe metal intercalates obtained by the ammonothermal method, the $T_{\mathrm{c}}$ is directly proportional to the interlayer distance up to a critical value of $d=$ 8.6 ̊ above which it starts decreasing and is dependent on the local structure of the $\mathrm{FeSe}_{4}$ tetrahedra as well as charge transfer to the FeSe layers. It is very difficult to compare the various results given the different nature and size of the intercalated metal, the quite wide spectrum of compositions and the diverse structural arrangements of the interlayer species. However, it appears that the general critical value does not apply in the case of Ba intercalates. The $d$-spacings observed throughout the series cross the $8.6 \AA$ boundary implying that Phase VI should display a higher $T_{\mathrm{c}}$ than Phase II. In addition, the interlayer distance reported for the ammonia free $\mathrm{Na}_{0.65} \mathrm{Fe}_{1.93} \mathrm{Se}_{2}$ is much shorter $(6.8 \AA)$ than in Phase VI but yet the $T_{\text {c }}$ is $3 \mathrm{~K}$ higher.

Recently, Ying et $a l^{5}$ have reported that by the ammonothermal method is possible to stabilize two distinct $\mathrm{ThCr}_{2} \mathrm{Si}_{2}$-type $\mathrm{K}$ intercalated superconducting phases which differ mainly in metal content, namely $\mathrm{K}_{0.3}\left(\mathrm{NH}_{3}\right)_{0.47} \mathrm{Fe}_{2} \mathrm{Se}_{2}$ and $\mathrm{K}_{0.6}\left(\mathrm{NH}_{3}\right)_{0.37} \mathrm{Fe}_{2} \mathrm{Se}_{2}$ with interlayer distance of 7.8 and $7.4 \AA$ and $T_{\mathrm{c}}$ of 44 and $30 \mathrm{~K}$ respectively. The drastic decrease in superconducting transition temperature is rationalized in terms of electron overdoping. Simple considerations of valence states would place the family of $\mathrm{Ba}$ intercalates and $\mathrm{K}_{0.6}\left(\mathrm{NH}_{3}\right)_{0.37} \mathrm{Fe}_{2} \mathrm{Se}_{2}$ at a similar doping level and both display a similarly enhanced $T_{\mathrm{c}}$ as compared to $\beta$-FeSe. A plot of $T_{\mathrm{c}}$ as function of interlayer spacing for these systems (Fig. 4 inset) shows a direct proportionality where $T_{\mathrm{c}}$ increases as $d$ becomes larger. This implies that in FeSe phases intercalated with large alkali and alkaline earth metals and comparable doping levels, the $T_{\mathrm{c}}$ depends mainly on the interlayer spacing which in turn is modulated by the amount of $\mathrm{NH}_{3}$ placed between the FeSe layers. It follows that the application of hydrostatic pressure to any of these systems will result in a reduction of the transition temperature. Indeed, it was recently shown that in $\mathrm{Cs}_{0.8}\left(\mathrm{NH}_{3}\right)_{y} \mathrm{Fe}_{2} \mathrm{Se}_{2} T_{\mathrm{c}}$ displays a negative pressure dependence. ${ }^{10}$

The work presented here highlights the importance of considering the size and chemical nature of the metal employed during the ammonothermal synthesis of superconducting $\beta$-FeSe intercalates. Metal content (and doping level) appears to be playing the decisive role in determining the value of the $T_{\mathrm{c}}$, which can then be further tuned by modulation of the interlayer spacing, in agreement with the conclusions of Guterding et al. $^{9}$

The Swiss-Norwegian Beam Line is thanked for the beamtime. This work is supported by the Norges Forskingsrådet project 214260 .

\section{Notes and references}

1 (a) H. Takahashi, K. Igawa, K. Arii, Y. Kamihara, M. Hirano and H. Hosono, Nature, 2008, 453, 376-378; (b) X. H. Chen, T. Wu, G. $\mathrm{Wu}, \mathrm{R} . \mathrm{H}$. Liu, H. Chen and D. F. Fang, Nature, 2008, 453, 761-762; (c) Zh.-A. Ren, W. Lu, J. Yang, W. Yi, X.-L. Shen, Zh.-C. Li, G.-C. Che, X.-L. Dong, L.-L. Sun, F. Zhou and Zh.-X. Zhao, Chin. Phys. Lett., 2008, 25, 2215-2216.

2 L. Malavasi and S. Margadonna, Chem. Soc. Rev., 2012, 41, 3897-3911.

3 D. P. Shoemaker, D. Y. Chung, H. Claus, M. C. Francisco, S. Avci, A. Llobet and M. G. Kanatzidis, Phys. Rev. B: Condens. Matter Mater. Phys., 2012, 86, 184511.

4 (a) L. Sun, X.-J. Chen, J. Guo, P. Gao, Q.-Zh. Huang, H. Wang, M. Fang, X. Chen, G. Chen, Q. Wu, Ch. Zhang, D. Gu, X. Dong, L. Wang, K. Yang, A. Li, X. Dai, H.-k. Mao and Zh. Zhao, Nature, 2012, 483, 67-69; (b) Y. Tian-Ping, W. Gang, J. Shi-Feng, Sh. Shi-Jie, Zh. Han, Zh. Ting-Ting, L. Xiao-Fang, W. Wan-Yan and Ch. XiaoLong, Chin. Phys. B, 2013, 22, 087412; (c) M. Burrard-Lucas, D. G. Free, S. J. Sedlmaier, J. D. Wright, S. J. Cassidy, Y. Hara, A. J. Corkett, T. Lancaster, P. J. Baker, S. J. Blundell and S. J. Clarke, Nat. Mater., 2013, 12, 15-19.

5 T. Ying, X. Chen, G. Wang, Sh. Jin, X. Lai, T. Zhou, H. Zhang, Sh. Shen and W. Wang, J. Am. Chem. Soc., 2013, 135, 2951-2954.

6 S. J. Sedlmaier, S. J. Cassidy, R. G. Morris, M. Drakopoulos, Ch. Reinhard, S. J. Moorhouse, D. O'Hare, P. Manuel, D. Khalyavin and S. J. Clarke, J. Am. Chem. Soc., 2014, 136, 630-633.

7 J. Guo, H. Lei, F. Hayashi and H. Hosono, Nat. Commun., 2014, 5, 4756.

8 T. P. Ying, X. L. Chen, G. Wang, S. F. Jin, T. T. Zhou, X. F. Lai, H. Zhang and W. Y. Wang, Sci. Rep., 2012, 2, 426.

9 D. Guterding, H. O. Jeschke, P. J. Hirschfield and R. Valenti, Phys. Rev. B: Condens. Matter Mater. Phys., 2015, 91, 041112(R).

10 L. Zheng, M. Izumi, Y. Sakai, R. Eguchi, H. Goto, Y. Takabayashi, T. Kambe, T. Onji, S. h. Araki, T. C. Kobayashi, J. Kim, A. Fujiwara and Y. Kubozono, Phys. Rev. B: Condens. Matter Mater. Phys., 2013, 88, 094521. 